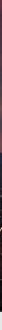

\title{
Conclusiones y futuro
}

\section{CARlos Santos Burguete \\ Centro Nacional de Predicción (CNP), Agencia Estatal de Meteorología (AEMET)}

\begin{abstract}
A large part of mathematics which becomes useful developed with absolutely no desire to be useful, and in a situation where nobody could possibly know in what area it would become useful; and there were no general indications that it ever would be so. By and large it is uniformly true in mathematics that there is a time lapse between a mathematical discovery and the moment when it is useful; and that this lapse of time can be anything from 30 to 100 years, in some cases even more; and that the whole system seems to function without any direction, without any reference to usefulness, and without any desire to do things which are useful.
\end{abstract}

The Role of Mathematics in the Sciences and in Society (1954) - JOHN VON NEUMANN

No se obtiene reconocimiento por predecir diluvios, sino por construir arcas.

LOU GERSTNER, CEO en IBM 1993-2002

Finalizamos esta aproximación a la moderna predicción del tiempo y proyección del clima resumiendo las principales ideas abordadas, respondiendo a las preguntas que se planteaban en el punto de partida $\mathrm{y}$, sobre todo, profundizando y ampliando las preguntas en un debate abierto que intenta, igual que los sistemas de predicción por conjuntos, acotar las incertidumbres que manejamos.

Palabras clave: física del caos, predicción meteorológica, modelos atmosféricos, predecibilidad, sistemas de predicción por conjuntos, predicción probabilista, proyecciones del clima, cambio climático, valor añadido del predictor, futuro de la predicción del tiempo.

Imagen parte superior: onda de montaña (altocumulus lenticularis duplicatus) sobre la sierra de Guadarrama vista desde el cerro del Tío Pío (Vallecas). En primer término la ciudad de Madrid. 21 de marzo de 2008 a las 19:34. Fotografía de José ANTONIO QUIRANTES Calvo. 


\subsection{Resumen de aspectos impor- tantes tratados y algunas con- clusiones}

Desde los albores de la humanidad, los factores ambientales han preocupado al ser humano, que ha intentado anticiparse a las inclemencias del tiempo y adaptarse a las mismas en la medida de lo posible. Hace relativamente poco tiempo, principios del siglo $\mathrm{XX}$ aproximadamente, que el enfoque científico dio una base sólida a la predicción meteorológica: la predicción del tiempo es reciente como ciencia o técnica de aplicación científica (cap. 3 en la página 21).

Las ciencias de la atmósfera ayudan a entender los procesos que tienen lugar en la misma mediante el método científico. En este libro hemos mostrado un pequeñísimo panorama resumido de la radiación, termodinámica del aire y la dinámica atmosférica (cap. 6 en la página 67)

Dos factores críticos han impulsado la ciencia de la predicción desde la mitad del siglo XX. El primero, es la aplicación de la supercomputación (cap. 11 en la página 145) para resolver aproximadamente las ecuaciones con las que describimos la atmósfera para realizar un simulación de su evolución. El segundo, es la observación cuantitativa del estado atmosférico, que desde el lanzamiento de los satélites meteorológicos (cap. 7 en la página 83) ha conseguido avances vertiginosos, aunque también los radares (cap. 8 en la página 103) y otras redes de observación son importantes (cap. 9 en la página 117, en el cual contamos algo de la ciencia de la climatología). Esos dos factores han revolucionado la predicción. Pero, aún hoy, estamos lejos de acertar siempre. ¿Por qué?

Profundizamos en estos puntos gracias a la teoría del caos (cap. 5 en la página 49 para los fundamentos, con una introducción histórica en el cap. 2 en la página 13). Como sistema dinámico, la atmósfera tiene un carácter fuertemente no lineal, con hipersensibilidad a las condiciones iniciales, lo que pone un límite físico a su predecibilidad (cap. 12 en la página 155). Profundizando en este aspecto, podemos decir que en el contexto de los sistemas dinámicos, las ecuaciones no lineales con las que describimos la evolución de la atmósfera tienen comportamiento caótico: cualquier incertidumbre en el proceso de predicción, como por ejemplo la diferencia entre dos condiciones iniciales ligeramente diferentes, puede crecer y conducir a dos estados de predicción muy diferentes. Como consecuencia, la denominada predecibilidad (cap. 12 en la página 155) asociada a la evolución de un estado atmosférico va a estar siempre limitada, en el espacio y en el tiempo, dependiendo en cada caso tanto de la magnitud de la incertidumbre como del propio estado atmosférico. Decimos entonces que la predecibilidad es dependiente del flujo. El meteorólogo EDWARD N. LORENZ estableció, en los años 60, el límite genérico de predecibilidad de la atmósfera en 2-3 semanas. De modo que los modelos atmosféricos deterministas (cap. 10 en la página 129) sufren estas limitaciones, que condujeron a investigar sus fuentes de error e incertidumbre.

Las fuentes de errores e incertidumbres que limitan la predecibilidad son principalmente: (1) inexactitudes en la medición del estado atmosférico inicial, estimado a partir de las observaciones disponibles, con su error observacional y su representatividad relativa, así como el error de los sistemas de asimilación; (2) imperfecciones en los modelos de predicción numérica del tiempo, relacionados con la formulación del modelo y las parametrizaciones físicas que describen los procesos submalla; y (3) los modelos Limited Area Model (LAM) heredan aproximaciones y errores de las condiciones de contorno que utilizan, provenientes de otros modelos de dominio mayor. Para muestrear adecuadamente el espacio de posibles estados atmosféricos, debemos tener en cuenta estas fuentes de incertidumbre y error mediante el uso de diferentes metodologías (caps. 16 en la página 243, 17 en la página 257 y 18 en la página 275). Algunos ejemplos de técnicas de muestreo de esos errores e incertidumbres descritos en este libro son, respectivamente, (1) Singular Vectors (SV), Bred Vectors (BV), Local Ensemble Transform Kalman Filter (LETKF) y Ensemble Data Assimilation (EDA); (2) multimodelo, multifísica o multiparametrizaciones, parametrizaciones estocásticas; y (3) multicondiciones de contorno.

Así pues, se hace necesario complementar a los modelos atmosféricos deterministas con sistemas que incluyan simulaciones sobre las incertidumbres, tanto en las condiciones iniciales como en el modelo. Este camino conduce a incluir la probabilidad de forma natural en nuestros sistemas predictivos. Las predicciones más fiables y hábiles a partir del estado observado actual de la atmósfera pueden obtenerse mediante una función de distribución de probabilidad (Probability Distribution Function, PDF, sec. 13.6.11 en la página 187) que describe, en teoría, un conjunto completo de posibles estados atmosféricos futuros. En la actualidad (2018), la única metodología plausible, técnicamente hablando, para construir una PDF prevista 
es la técnica de sistema(s) de predicción por conjuntos (SPC) o ensembles (cap. 13 en la página 165): se genera una muestra de predicciones de modelos atmosféricos de predicción numérica del tiempo, diferentes pero igualmente plausibles todos ellos, a los que se denomina miembros del conjunto. Estos SPC han demostrado ser más fiables (fiabilidad, sec. 15.9.4 en la página 226) y hábiles (habilidad o skill, sec. 15.2.4 en la página 212), en el espacio de las probabilidades, que un sólo modelo determinista, aunque éste pueda disfrutar de mayor resolución. Como mínimo, los SPC son un complemento indispensable para los modelos deterministas y, poco a poco, el enfoque probabilista va adquiriendo protagonismo. Hay una serie de aspectos fundamentales que podemos destacar de los SPC, algunos de ellos relacionados entre sí:

- Podemos simular el efecto de las diversas fuentes de error en la predicción.

- Nos permiten estimar la PDF (sec. 13.6.11 en la página 187) de los estados de predicción.

- Proporcionan diferentes escenarios atmosféricos futuros y permiten al usuario estimar la probabilidad de esos escenarios.

- Nos dan información explícita, cuantitativa y detallada sobre la dispersión atmosférica, que está muy relacionada con la incertidumbre y, por ende, con la predecibilidad.

- Permiten identificar áreas y/o alcances predictivos con baja predecibilidad.

- La predecibilidad es dependiente del flujo. Podemos, con los SPC, calibrar esa dependencia, encontrando relaciones entre la predecibilidad y los patrones atmosféricos (distintas situaciones) con los que trabajamos.

- Nos permiten evaluar el riesgo potencial de fenómenos adversos. Predecir la predecibilidad es predecir el riesgo meteorológico y climático, un elemento esencial en cualquier sistema práctico cuantitativo de predicción, especialmente en lo referente a estos fenómenos adversos.

- El promedio del ensemble no es necesariamente una situación meteorológica, ni debe ser tomado como escenario atmosférico plausible.

- Los productos para la predicción que proveen los SPC representan su carácter inherentemente probabilista.

- Los SPC se empezaron a utilizar en predicción operativa en la Agencia Estatal de Meteorología (AEMET) durante los años 90 y son una poderosa herramienta, tanto para la predicción a escala sinóptica como, recientemente con el desarrollo de SPC kilométricos, para la predicción a mesoescala.

La calidad y el valor de los SPC puede evaluarse objetivamente mediante procedimientos de verificación probabilista (cap. 15 en la página 207), que permiten contrastar una serie de propiedades. La consistencia estadística con las observaciones a gran escala puede ser evaluada mediante histogramas de rango o con la relación dispersión-error. La respuesta frente a eventos binarios (e. g. llueve o no llueve) se plasma en varias propiedades: la agudeza (sec. 15.9.3 en la página 225) representa la tendencia a dar probabilidades bajas o altas pero no intermedias; la fiabilidad (sec. 15.9.4 en la página 226) mide la consistencia entre probabilidades previstas y las correspondientes frecuencias condicionales de observación; la resolución probabilista indica el grado de discernimiento entre distintas probabilidades con respecto a la climatología muestral; la habilidad (skill, sec. 15.2.4 en la página 212) es una medida de calidad relativa con respecto a un sistema predictivo de referencia, como pueden ser la persistencia, la climatología muestral o algún sistema predictivo con el que comparar. Estas propiedades pueden verse gráficamente en los diagramas de atributos, así como en los índices de BRIER o Brier Skill Score. La discriminación mide la capacidad del sistema para discernir entre la ocurrencia o no ocurrencia del evento, representándose en la denominada curva ROC y midiéndose con el índice Área bajo la curva ROC. El valor económico relativo puede también evaluarse gráfica y numéricamente, mostrando el valor de usar el sistema predictivo con respecto a otros sistemas y, por tanto, mostrando la rentabilidad de estos SPC. La verificación, además de medir el rendimiento de las predicciones orienta, tanto a los modelizadores como a predictores y usuarios finales, para mejorar tanto los modelos como los SPC y sacar máximo provecho de los mismos.

Antes de utilizar directamente las salidas de los SPC $\mathrm{y}$, con las potentes herramientas de verificación de disponibles, es conveniente hacer pasar al sistema por un proceso de calibración estadística (cap. 14 en la página 193), para filtrar los errores sistemáticos en la medida de lo posible. Algunas técnicas presentadas aquí son el promediado bayesiano de modelo (Bayesian Model Averaging, BMA) y las regresiones Logística y Logística Extendida (Logistic Regression, LR and Extended Logistic Regression, ELR). A pesar de que estas técnicas mejoran ostensiblemente el rendimiento estadístico del sistema en periodos de tiempo determinados, los fenómenos extremos (entre los cuales encontramos numerosos fenómenos adversos) siguen 
siendo un problema: el proceso de calibración tiende a suavizar las salidas de los SPC, de modo que se les suele escapar la señal del fenómeno extremo cuando éste va a tener lugar. Ésta es actualmente una necesaria línea de investigación.

Hemos intentado mostrar, sabiendo que siempre nos quedaríamos cortos, una serie de SPC representativos del abanico de escalas en el espacio y en el tiempo, a la par que de varios ámbitos diferentes (parte IV en la página 288). El SPC global del European Centre for Medium-range Weather Forecasts -Centro Europeo de Predicción a Plazo Medio- (ECMWF) (cap. 19 en la página 289) y el regional europeo Grand Limited Area Model Ensemble Prediction System (GLAMEPS) del consorcio High Resolution Limited Area Model (HIRLAM) (cap. 20 en la página 301). Dentro de los desarrollados en España, el de escala sinóptica AEMET-SREPS (cap. 21 en la página 313) y el moderno y mesoescalar AEMET- $\gamma$ SREPS (cap. 22 en la página 333). Como ejemplos en otros países, hemos descrito brevemente los MOGREPS de Reino Unido (cap. 23 en la página 361), los SPC experimentales de México (cap. 24 en la página 367). Para no quedarnos sólo en la atmósfera, el proyecto MyWave (cap. 25 en la página 373) nos ha permitido mostrar SPC de otros ámbitos, en este caso el oleaje. En el capítulo hacemos una ronda más amplia, pero también más esquemática, para poder mostrar una pincelada de otros SPC operativos en otras partes del mundo.

La aplicación directa de los SPC es lo que denominamos predicción probabilista (cap. 27 en la página 401). Hemos presentado una buena cantidad de productos probabilistas para la predicción operativa que, como decíamos, reflejan la naturaleza, a su vez probabilista, de los SPC. Permiten, por tanto, trabajar con el concepto de predecibilidad y sirven como guía probabilista de predicción. Entre otros, están: mapas de dispersiónpromedio, agrupamiento, mapas de probabilidad y de percentiles, EPSgramas, Índice de Predicción Extrema o Extreme Forecast Index (EFI) y, para terminar, las trayectorias de ciclones tropicales.

Hay numerosas aplicaciones, además de la predicción probabilista aplicada a la predicción del tiempo. La observación de la atmósfera, cuando está sujeta a incertidumbres, puede describirse mediante técnicas parecidas y ponemos como ejemplo la observación radar en el capítulo 30 en la página 517. La predicción aeronáutica, de valor incalculable para la aviación comercial y militar, puede disfrutar de las ventajas de los SPC de altísima resolución que permiten elaborar pronósticos de aeródromo con completísimos muestreos de incertidumbres (cap. 31 en la página 523). La aplicación de SPC en entornos específicos nos importa especialmente pues en España tenemos, entre otras, el área mediterránea, que con su intrincada geografía y la capacidad calorífica de un mar casi cerrado, lo convierten en un entorno endiabladamente poco predecible. Es en estos entornos difíciles donde los SPC pueden dar su do de pecho. Dedicamos un capítulo especial al Mediterráneo (cap. 32 en la página 531) y uno a un ejemplo concreto en ese entorno, los mediterranean hurricanes o medicanes (cap. 33 en la página 551). Incluyendo la previsión de medicanes, de rayos (cap. 34 en la página 563) y algunos otros, los fenómenos adversos han merecido nuestra atención especial: son aquellos de más impacto socioeconómico. Por otro lado, los fenómenos extremos, aquellos que por su rareza están en las colas de las distribuciones climatológicas, se conocen poco. Los fenómenos extremos no siempre son adversos y, viceversa, hay fenómenos adversos que no son extremos. Examinamos una herramienta operativa para la gestión de avisos de fenómenos meteorológicos adversos. No podían faltar las energías sostenibles, como la solar (cap. 38 en la página 599) y la eólica (cap. 39 en la página 607), ámbitos en los que los SPC pueden aportar un valor económico que, por cierto, puede medirse cuantitativamente (sec. 15.10 en la página 234). Mostramos las aplicaciones de los SPC en otros países hermanos, como siempre quedándonos cortos, mostrando al menos dos: Argentina (cap. 37 en la página 583) y Portugal (cap. 36 en la página 579).

Los SPC se utilizan en la actualidad (2018) en casi todas las escalas, desde la mesoescala hasta las proyecciones climáticas. Ambos extremos definen problemas de predecibilidad diferentes, desde el punto de vista teórico: la predicción del tiempo es un problema de valor inicial y las proyecciones del clima un problema de condiciones de contorno (sec. 12.2 en la página 157 y sec. 12.2.3 en la página 158). En el camino de menor a mayor escala, pasamos por la predicciones de largo plazo, mensual, estacional, anual y decadal (cap. 28 en la página 447), hasta llegar, finalmente, a las proyecciones del clima (cap. 29 en la página 477) donde hablamos de abanicos de incertidumbres específicos para esas escalas. Ahí, el papel de los SPC es crucial para el muestreo y acotación de incertidumbres. De ese modo podemos hablar de calentamiento global antropogénico con una base científica.

Hemos dedicado una parte a algunos aspectos relacionados con la sociedad. La percepción y entendimiento 
de los conceptos relacionados con la probabilidad, la comunicación de la información meteorológica de carácter probabilista y el reto que todo ello supone para los medios, es un aspecto crucial abordado en el capítulo 40 en la página 615. Si la sociedad demanda predicciones mejores, los SPC las pueden dar, pero los servicios meteorológicos tienen que abrirse a esa posibilidad, un proceso que no siempre lleva el ímpetu necesario. El programa europeo Meteoalerta de gestión y coordinación de avisos de fenómenos meteorológicos adversos se describe con un cierto detalle (cap. 41 en la página 627), así como el proyecto PROFORCE de aplicación de SPC para Meteoalerta (cap. 42 en la página 633). En esta parte añadimos un capítulo que llama la atención sobre los malentendidos en la comunicación de la información meteorológica por un uso inadecuado de la terminología (cap. 43 en la página 639).

La aplicación directa de la predicción probabilista como técnica de predicción del tiempo se expone en la parte de casos (parte VII en la página 654), que aprovechamos para revisitar algunos casos notorios o históricos, algunos más cotidianos, relacionados o no con fenómenos meteorológicos adversos. El récord histórico de precipitación diaria en España de Oliva-Gandía 1987 se aborda en el capítulo 44 en la página 655 , con una descripción de notable interés histórico y meteorológico, acompañada con la aplicación de modernos SPC y predicción probabilista. Sobre predicción de aludes hay poca bibliografía en castellano; intentamos así en el capítulo 45 en la página 689 cubrir dos huecos: el de previsión de aludes y el de hacerlo con predicción probabilista. En el capítulo 46 en la página 717 mostramos dos casos de ciclones tropicales atlánticos: Ike y Álex, cuya previsión es probabilista (por la cuenta que trae a los países afectados); con este capítulo salimos del ámbito geográfico de España. Y seguimos fuera de España en el capítulo 47 en la página 729 , donde realizamos un ejercicio de predicción probabilista de largo plazo, ordinaria, en Myanmar. Volvemos a España con la influencia de la tormenta tropical Gastón en una ola de calor que tuvo lugar en septiembre de 2016 (cap. 48 en la página 741). Las lluvias torrenciales en el Maresme, Barcelona, en octubre de 2016 ocupan el capítulo 49 en la página 753. Poco después, en diciembre de 2016, tuvo lugar un temporal de Levante que azotó el Mediterráneo, explorado en el capítulo 50 en la página 763. Ese mismo caso, utilizando otras herramientas y contrastándolo con las precipitaciones, también intensas, de marzo de 2017, se expone en el capítulo 51 en la página 775 . En enero de 2017 otro temporal dejó nieve en localidades como Torrevieja, donde hacía 103 años que no nevaba (cap. 52 en la página 795). Una borrasca con baja predecibilidad, en febrero de 2017, fue un caso especialmente difícil (cap. 53 en la página 805). En ese mismo mes tuvo lugar un temporal marítimo en Galicia de bastante impacto (cap. 54 en la página 815). Otra vez en el mismo mes, se dieron lluvias torrenciales en Málaga, con un fuerte impacto socioeconómico en el área metropolitana (cap. 55 en la página 827). Dedicamos a Canarias tres casos reunidos en el capítulo 56 en la página 839 , tanto de lluvias como de vientos intensos. El fenómeno de la nieve lo abordamos con una predicción probabilista ordinaria de un caso de posibles nevadas generalizadas en la Península (cap. 57 en la página 855$)$. Otra predicción, ordinaria desde el punto de vista técnico, pero crucial desde el social, es la de Semana Santa de 2017 (cap. 58 en la página 867). Cerramos esta parte con una depresión aislada de niveles altos (DANA) de principio de verano de 2017 (cap 59 en la página 879).

\subsection{Algunas respuestas a las pre- guntas del principio}

En la introducción de este libro comenzábamos formulando algunas preguntas habituales que la sociedad plantea a la comunidad meteorológica, en relación con la predicción del tiempo y con el candente tema del cambio climático. Uno de los objetivos de este libro es dar algunas respuestas a esas preguntas o, si las respuestas no son fáciles o no son definitivas, al menos ampliar un poco la perspectiva en estos temas para, tal vez, encontrar dentro de las preguntas conceptos interesantes con los que ampliar nuestro conocimiento. Anticipábamos entonces un esbozo de las respuestas y, ahora, una vez recorrido, al menos en parte, el camino del libro podemos expresar las respuestas con mucho más detalle y, seguramente, ampliar el abanico de preguntas. Hemos intentado que las respuestas sean, hasta cierto punto, autodefinidas $y$, aunque enlacen a algunos capítulos del libro, no se enlacen excesivamente unas a otras. El resultado es, inevitablemente, cierta redundancia en las respuestas, redundancia por la que pedimos disculpas de antemano a quien quiera seguir secuencialmente todas ellas. Añadimos, a las preguntas iniciales, algunas otras que también pueden resultar interesantes y completar algunas de las ideas esbozadas. 
¿Hasta cuántos días son fiables las predicciones del tiempo?. A la luz de los conceptos alumbrados en el libro, ya sabemos que la mejor respuesta, aunque pueda sonar jocoso, es «depende». En el capítulo 15 en la página 207 hemos visto el concepto de límite de predecibilidad (sec. 15.3 en la página 216) y cómo gracias a él puede darse una respuesta aproximada a esta pregunta, respuesta válida tanto para los científicos como para la sociedad abierta.

El límite de predecibilidad es el horizonte más largo en el que se puede dar una predicción con un mínimo de calidad, midiendo esa calidad mediante parámetros concretos como son el coeficiente de correlación de anomalías, que miden de algún modo la consistencia entre la predicción y la posterior observación. Ese límite de predecibilidad es dependiente del flujo (sec. 27.10 en la página 434), es decir, depende de la situación atmosférica: cuando el tiempo es anticiclónico el límite de predecibilidad puede prolongarse hasta más de diez días mientras que, en una situación muy inestable como puede ser una pequeña borrasca de desplazamiento rápido, el límite de predecibilidad puede ser inferior a 24 horas. De modo que cuando se da un límite de predecibilidad general correspondiente a un año en una región grande del planeta, se trata de algún promedio o ponderación y, por lo tanto, una generalización, a menudo en el margen de la validez.

De cualquier modo, podemos resumir la respuesta diciendo que, en latitudes medias (por ejemplo, Europa), la predecibilidad de la atmósfera ha venido mejorando a razón de un día (o un día y medio, según el parámetro) por década. Por ejemplo, nuestras predicciones de la dinámica atmosférica (altura geopotencial en $500 \mathrm{hPa}$ ) eran fiables (un coeficiente de correlación de anomalías de por lo menos un $80 \%$ ) hasta tres días y medio (D+3.5) a principios de los años ochenta y, ahora en 2018, son fiables hasta algo más de unos seis días y medio (D+6.5). Estas pautas pueden examinarse con detalle en las Figuras 15.7 en la página 215 y 15.2 en la página 209. La precipitación o el viento son más difíciles de predecir, pero esta pauta orientativa puede aplicarse de un modo similar.

¿En qué se basa la predicción por localidades?. Las predicciones del tiempo se apoyan básicamente en modelos atmosféricos, que son complejos programas de ordenador, alimentados por datos observados de la atmósfera, sobre todo de satélite. Estos modelos proveen a los meteorólogos predictores o a usuarios finales de una primera aproximación al estado futuro de la atmósfera. Como representaciones de la realidad, no son perfectos y el predictor corrige, añade y, en general, mejora la predicción del ordenador. Los citados modelos trabajan dividiendo el planeta en una malla o algo similar, con cajitas (gridbox) y resolviendo las ecuaciones de la física en cada una de ellas, de modo que cuanto más cerca estemos del centro de una cajita más fiable será, en principio, la predicción.

La predicción por localidades se realiza con un programa aparte que interpola los datos desde el centro de la cajita o cajitas más cercanas al punto de la localidad de interés. Ese proceso acentúa la imperfección del modelo, introduciendo ruido. Por tanto, las predicciones por localidades deben consultarse con precaución, sobre todo si se trata de localidades o puntos en lugares montañosos o costeros.

\begin{abstract}
¿Pueden hacerse predicciones con seis meses de antelación?. El meteorólogo EDWARD N. LORENZ anticipó en 1963 que existe un límite superior en la predecibilidad de la atmósfera, que estableció en 2-3 semanas. Más adelante, en la década de los 90, científicos como EUGENIA KALNAY introdujeron de forma natural la incertidumbre en el proceso de predicción numérica del tiempo, poniendo en marcha los primeros sistemas de predicción por conjuntos, que permiten, entre otras muchas ventajas, evaluar la predecibilidad atmosférica.
\end{abstract}

Tal como entiende la sociedad las predicciones del tiempo, de modo que informen con claridad y cierta exactitud sobre la temperatura, precipitación, viento que podemos esperar, las predicciones sólo pueden hacerse hasta un límite de unos quince días en el mejor de los casos (tiempo anticiclónico).

Más allá empieza otro juego distinto, en el que la exactitud da paso a la estadística y a las pautas generales. En las llamadas predicciones mensuales y estacionales, los centros meteorológicos podemos anticipar ciertas pautas generales, expresadas en términos de cercanía o alejamiento de la normalidad, normalidad expresada como promedio de los últimos 20-30 años. Así, podemos anticipar que el mes de mayo será más lluvioso de lo normal y más cálido de lo normal, pero anticipar qué sucederá el 15 de mayo o el 25 de mayo con exactitud no es posible. Y así sucesivamente, cuanto más lejano el horizonte predictivo, más difuminada será la predicción. Las predicciones decadales son aún más difusas que las anuales. 
¿Cómo puede predecirse el cambio climático?. Continuando el hilo de la respuesta anterior sobre si era posible predecir el tiempo con seis meses de antelación, recordemos que la respuesta es no, en los términos habituales. La predicción estacional se expresa en términos de pautas. Las llamadas proyecciones climáticas suponen unos cuantos pasos más allá en ese mismo razonamiento.

El ejemplo del dado ilustra este concepto: al tirar un dado, no puedo predecir el número que va a salir en cada tirada, pero sí que puedo predecir estadísticas de una serie grande de tiradas. Puedo saber que, si tiro el dado 600 veces, saldrán aproximadamente (y si el dado no está trucado), 100 unos, 100 doses, etc. Las proyecciones climáticas funcionan de este modo. Nada podemos saber sobre lo que pasará el 26 de julio de 2100. Sin embargo, podemos adelantar cómo aumentarán la temperatura y el nivel medio del mar en los próximos 100 años, dentro de un abanico de incertidumbre que también conocemos.

Los modelos usados para proyecciones climáticas son, esencialmente, similares a los usados en predicción del tiempo, aunque el problema que han de resolver es de diferente naturaleza. En términos de ecuaciones diferenciales o en diferencias, la predicción del tiempo es un problema de valor inicial: las condiciones iniciales son cruciales para la evolución del estado del sistema. Las proyecciones del clima, sin embargo, son un problema de forzamiento o condiciones de contorno: cuánto $\mathrm{CO}_{2}$ vamos a seguir liberando, etc. En ambos casos hay una herramienta común: los SPC, que permiten evaluar las incertidumbres asociadas a ambos procesos.

\section{¿Se harán predicciones cada vez más exactas y pre-} cisas?. ¿Pueden realmente hacerse predicciones cada vez más precisas y a la vez exactas? En el sentido tradicional y, por tanto, determinista, del concepto de predicción, la respuesta es no. Se ha constatado en el libro varias veces y en esta conclusión también, que hay un límite de predecibilidad para la atmósfera. Los modelos atmosféricos han mejorado y seguirán mejorando, gracias a los avances científicos en meteorología y a los avances tecnológicos en satélites y supercomputación, de modo que hacen predicciones con una resolución más fina (más precisas) y con niveles de exactitud al menos iguales a los anteriores (exactas). Pero hay límites físicos para esa progresión, debidos fundamentalmente al carácter caótico de las ecuaciones subyacentes con las que describimos la atmósfera y que sirven de base a las simulaciones computacionales que hacemos de la misma.

Ahora bien, esa limitación fundamental no impide que, en otro sentido, las predicciones puedan mejorar. El advenimiento de los SPC es un claro ejemplo de cómo pueden hacerse predicciones «mejores», dado que no pueden ser más «exactas». La introducción de la probabilidad como ingrediente natural del proceso de predicción es una muestra de crecimiento conceptual en una ciencia-técnica como la predicción meteorológica; en cierto modo, una ciencia más sana, porque ha sabido reconocer sus limitaciones y, más allá de eso, asumirlas y crecer en otras direcciones. Si los avances tecnológicos y los científicos siguen siendo respaldados por la sociedad, el crecimiento está garantizado, aunque no siempre sea en la dirección pensada inicialmente.

¿Se usa la teoría del caos en la predicción del tiempo?. Sí. Los SPC se fundamentan en los estudios sobre la predecibilidad de la atmósfera (cap. 12 en la página 155), los sistemas dinámicos no lineales (cap. 5.1.5 en la página 54), su sensibilidad a las condiciones iniciales y a la formulación del modelo.

Los sistemas caóticos son un subconjunto de los sistemas no lineales. La problemática de la predecibilidad de la atmósfera es un caso de caos determinista. Conocemos las ecuaciones que describen la evolución del estado atmosférico, sabemos que son deterministas y que, en teoría, tienen solución única. Pero, dada la naturaleza caótica de las mismas, en la práctica es imposible encontrar una solución exacta, pues sufren de hipersensibilidad a las condiciones iniciales: Dadas dos condiciones iniciales muy similares, con una diferencia muy pequeña entre ambas y tomando esas condiciones como puntos de partida del estado atmosférico, la diferencia entre sus evoluciones deja de ser pequeña y puede amplificarse mucho.

La misma hipersensibilidad se da con la formulación del modelo: pequeñas variaciones en parámetros del mismo pueden conducir a grandes diferencias en el resultado. Este problema pone un límite físico a la predecibilidad de la atmósfera, límite que el meteorólogo EDWARD N. LORENZ anticipó en 1963 y estableció en 2-3 semanas. En este sentido, estos descubrimientos relacionados con la física del caos fueron aplicados más tarde, en la década de los 90, por científicos como EUGENIA KALNAY, introduciendo de forma natural la incertidumbre en el proceso de predicción 
numérica del tiempo, poniendo de este modo en marcha los primeros SPC, con los que puede evaluarse la predecibilidad atmosférica.

¿Qué es el efecto mariposa?. El efecto mariposa se relata con mucho detalle y profundidad en el capítulo 5 en la página 49, en particular en la sección 5.5 en la página 64. Se trata de un símil atribuido al meteorólogo EDWARD NORTON LORENZ que acuñó la frase «El aleteo de una mariposa en Brasil puede producir un tornado en Texas». Anteriormente, se había referido a una gaviota provocando una tormenta [9].

La frase no es rigurosamente cierta desde el punto de vista científico, pero resume de manera visual el fenómeno denominado sensibilidad a las condiciones iniciales: los sistemas dinámicos llamados no lineales, de los cuales la atmósfera es un ejemplo, sufren esta sensibilidad. Cuando se simulan mediante ordenador, pequeñas variaciones en las condiciones iniciales usadas, pueden convertirse en grandes variaciones en la evolución del sistema.

A la postre, este problema pone un límite superior a la denominada predecibilidad atmosférica. LORENZ no fue el primero en utilizar un símil de este estilo para ilustrar la limitación en la predecibilidad de la atmósfera o, tampoco, en preocuparse por el problema. Anteriormente, NORBERT WIENER en los años 50 ya expresaba su preocupación [11] y, a finales del siglo XIX, WILLIAM SUDDARDS FRANKLIN escribió [9], metafóricamente, que el vuelo de un saltamontes en Montana podría trasladar una tormenta de Filadelfia a Nueva York. De todos modos, se atribuye a LORENZ la idea del efecto mariposa. Dicho efecto es ideal para ilustrar numerosos conceptos relacionados con la predecibilidad, véase e. g. el capítulo 12 en la página 155.

¿Si tuviéramos condiciones iniciales perfectas podríamos evitar el problema del caos determinista y traspasar el límite de predecibilidad de la atmósfera?. Es una idea que tiene cierta lógica interna, apta más para una novela o película que para llevarla realmente a cabo. Para empezar, nos encontramos con el resbaladizo adjetivo de perfectas al referirse a las condiciones iniciales perfectas. Defina usted perfectas. Por supuesto que es una idea muy natural y «perfectamente» definida, sobre todo en nuestras mentes.

Pero intentemos hacer una figuración realista de esta idea. Tal vez lo más adecuado para hablar de perfección sea una combinación de precisión y exactitud: precisión es el grado de detalle con el que podemos describir los fenómenos; si éstos son medibles, entonces sería el número de cifras significativas para medir, por ejemplo, las distancias. Por otro lado, exactitud se refiere a la diferencia con la realidad; cuanto más exacto, menor es esa diferencia con el valor real. Entonces, condiciones iniciales perfectas serían aquellas con una precisión lo más alta posible y con una exactitud perfecta. Pero, en última instancia, ambas concepciones se plasman en un número significativo de cifras: nuestra capacidad de medir. Para medir el estado inicial de la atmósfera, mediríamos presión, temperatura, humedad, viento, variables todas ellas con una cierta precisión y exactitud en los instrumentos que, directa o indirectamente, están midiendo distancias internas en procesos indirectos.

En cualquier caso, esa significación en la precisión y la exactitud va siempre asociada a una escala: en esa cierta escala, la precisión y exactitud serán perfectas. Pero siempre existirá una escala más fina en la que no sean perfectas. Todo este razonamiento tan teórico, en la práctica puede expresarse en términos de modelos atmosféricos: los modelos sinópticos han ido dejando paso a los mesoescalares y, en la historia de los modelos, diferentes escalas se han ido simulando cada vez mejor. Pero cuando conseguimos simular extraordinariamente bien una escala, por ejemplo, la sinóptica, entonces pondremos nuestra meta en simular igual de bien la mesoescala atmosférica. $\mathrm{Y}$ así sucesivamente. En ese sentido, no habrá nunca condiciones iniciales perfectas: siempre habrá una escala más fina, impensable en el pasado, en la que se puede mejorar.

¿Pero en un futuro se podrá traspasar el límite de predecibilidad, verdad?. Decía ARTHUR C. CLARKE en su obra Perfiles del futuro que cualquier manifestación científica o tecnológica suficientemente avanzada para su época es indistinguible de la magia. Si pensamos, guiados por la intuición y el sentido común, que a principios del siglo XX no existían la mayoría de adelantos tecnológicos que disfrutamos hoy en día, como por ejemplo la telefonía móvil, la radio y la televisión, los automóviles y aviones, los ordenadores, internet, etc., entonces podrían ser posibles en un futuro otras ensoñaciones del ser humano como la telepatía, el teletransporte, la telequinesis o la inmortalidad. Es difícil rebatir argumentos de este estilo, al no prestar atención a los fundamentos científicos en sí mismos.

Si se les presta atención, las limitaciones son más severas. Piénsese, por ejemplo, en el Principio de incer- 
tidumbre de HEISENBERG: es una limitación natural a la que, en principio, la tecnología de toda época estará sujeta. ¿o no? La tecnología puede evolucionar, pero la ciencia también. En este punto el debate se abre tanto que no podemos, realmente, anticipar mucho. Lo que sí podemos decir es que, según tal principio, la medida simultánea de posición y momento de las partículas elementales no puede realizarse y, por tanto, siempre que la ciencia no cambie, cualquier adelanto tecnológico tendrá que realizarse respetando esa limitación.

En un sentido análogo podemos lanzar vaticinios sobre el límite de predecibilidad de la atmósfera que, además, no está sujeto a un principio tan precisamente definido como el de Heisenberg. Podemos decir que, si las simulaciones de la atmósfera se siguen realizando con los esquemas similares a los actuales, aunque se avance en resolución de los modelos y en calidad de los satélites, etc., la atmósfera tendrá siempre limitada su predecibilidad.

\subsection{Conclusiones de la experien- cia en el desarrollo de SPC}

Una parte de los autores de este libro han participado o participan, directa o indirectamente, en la investigación y desarrollo de SPC en AEMET. La experiencia del Grupo de Predecibilidad así llamado y algunas otras actividades, tanto en la gestación de sistemas puestos en operación como la participación en foros internacionales, ha ido arrojando algunas conclusiones sobre decisiones científicas y técnicas más o menos acertadas, que plasmamos a continuación.

Multimodelo y multianálisis frente a un solo modelo. Nuestra experiencia en AEMET y el grupo HIRLAM en investigación y desarrollo de SPC con las técnicas multimodelo (sec. 13.5 en la página 174), usando varios modelos LAM y multianálisis (sec. 13.5 en la página 174), procedentes de diferentes modelos Global Circulation Model (GCM), han mostrado mejor rendimiento que otras aproximaciones basadas en un solo modelo. Esto demuestra que aún no tenemos conocimiento suficiente sobre las diferentes incertidumbres del modelo y que aún podría haber otras fuentes de error.

En principio, el uso combinado de diferentes metodologías de muestreo de errores e incertidumbres parece dar los mejores rendimientos, al estar muestreando el espacio de fases de un forma algo más exhaustiva. Gran parte de los SPC actuales padecen de subdispersión, problema que podría subsanarse con estas combinaciones de tećnicas, teniendo siempre cuidado en aumentar no sólo la dispersión, sino también la calidad, para mantener el balance dispersión-error (sec. 15.8.2 en la página 223) adecuado. Otra fuente indudable de mejora viene de los progresos en asimilación de datos (sec. 10.3 en la página 133 y cap. 16 en la página 243): por ejemplo, en el ECMWF, poco a poco la tećnica de asimilación de datos por conjuntos (EDA, sec. 16.2.5 en la página 248) va sustituyendo a la técnica de vectores singulares (sec. 16.2.6 en la página 249).

Hay que señalar también que nuestra experiencia con la técnica multimodelo nos ha permitido aprender algo del folklore que significa poner en operación un sistema de esta envergadura: muchos modelos pueden usarse para investigación, pero no pueden usarse para poner en operación un sistema predictivo; en ocasiones hay que pagar licencias y no siempre hay claridad suficiente al respecto de las mismas. Esta problemática nos condujo, en esta segunda época coincidente con la escala convectiva, a utilizar desde el principio solamente modelos de los que tuviéramos licencia por ser miembros del consorcio correspondiente (e. g. HARMONIE-AROME) o que fuesen completamente libres (e. g. WRF).

Impacto del tamaño del ensemble o número de miembros. Durante los primeros años de desarrollo de diferentes SPC por diversas partes del mundo, esta cuestión generó numerosos e interesantes debates que, aunque a día de hoy no tengan una respuesta teórica definitiva, sí que se tiene una pauta práctica fundamentada en resultados y rendimientos. Yendo aún más profundo en los aspectos teóricos, esta cuestión está relacionada con otra más profunda que detallamos después: ¿si la PDF (sec. 13.6.11 en la página 187) supuesta del estado atmosférico es continua y nosotros la muestreamos con un conjunto discreto, entonces estamos seguros de realizar un muestreo incompleto, no es así? Sin llegar necesariamente tan hondo, el asunto del número de miembros óptimo para un SPC se ha debatido desde muchos ángulos y expondremos aquí las conclusiones más relevantes. A bote pronto, cuantos más miembros, mejor: más próxima estará la PDF simulada a un continuo.

Ahora bien, la primera y más importante limitación es la de recursos computacionales: resulta mucho más caro integrar 1000 veces el modelo que integrarlo 50. 
Por otro lado, integrarlo sólo 5 veces parece, intuitivamente, un muestreo pobre de los posibles estados atmosféricos. Los grandes SPC del mundo, e. g. el GEFS del NCEP con 21 miembros y el ECENS del ECMWF con 51 miembros, han apostado por un orden de magnitud similar, en torno a 20-50 miembros, cantidad dada por la experiencia respectiva. En términos prácticos enunciados conjuntamente por desarrolladores y usuarios de predicción probabilista, podemos afirmar que a partir de un número que oscila entre 50 y 200 , las mejoras en calidad, habilidad (skill) o valor llegan a saturarse, de modo que invertir en más recurso computacional a partir de 50 miembros parece no ser rentable.

No obstante, el punto crítico lo ponen los fenómenos extremos (que no siempre son adversos y viceversa): aquellos fenómenos que están en la cola de la distribución climatológica, cuando vayan a tener lugar, a un SPC de 10 miembros probablemente se le escapen, cosa que no sucederá con un SPC de 100 miembros. En estos casos, sólo 1 ó 2 miembros de un SPC de 51 como el del ECMWF son los que vislumbran un escenario extremo. Y puede ser suficiente para poner sobre aviso a los predictores o usuarios finales del SPC. Esa es una de las claves. En el JMA apuestan por ensembles de gran tamaño, tienen muchos recursos computacionales para ello.

Papel de la resolución. Durante esta primera era de crecimiento de este paradigma, grandes centros como el ECMWF o el NCEP han dividido sus recursos computacionales más o menos a la mitad: $50 \%$ para el modelo determinista de mayor resolución y otro $50 \%$ para el ensemble, que funcionaba con una resolución inferior, tradicionalmente con un tamaño de celda de aproximadamente el doble que el del determinista.

En relación con el número de miembros óptimo descrito anteriormente, algunas personas se preguntan por qué no usar un SPC con resolución equiparable a la superior del modelo determinista, bajando si es preciso el número de miembros? La resolución es una característica ligada a la calidad determinista de los miembros. El número de miembros va ligado a la calidad probabilista, a la capacidad de exploración del espacio de fases de la atmósfera. El número de miembros no se va a reducir, al menos en principio, en los grandes centros dedicados a la predicción por conjuntos. Ahora bien, el ECMWF ha anunciado en su estrategia para la década 2015-2025, que volcará sus recursos en el SPC, en la predicción probabilista.
Esto no quiere decir que se abandone el desarrollo del modelo determinista en absoluto: el modelo determinista es el ingrediente fundamental del ensemble y siempre será imprescindible la investigación y el desarrollo en el mismo.

Por otro lado, un buen modelo determinista es condición necesaria pero no suficiente para un buen SPC. Lo que sí puede cambiar es el balance en los recursos computacionales dedicados a uno y a otro. En cualquier caso, en la próxima década tendrá lugar otro aumento crítico en las resoluciones horizontal y vertical de los SPC ligado al de los modelos correspondientes. En los SPC regionales ya hemos traspasado la barrera de los modelos no hidrostáticos, e. g. con AEMET- $\gamma$ SREPS, y esa barrera se traspasará pronto para los SPC globales.

En los SPC regionales, que a menudo están ya en la escala meso-gamma (cloud-resolving) a 1-4 km, es probable que se llegue a resoluciones inferiores al kilómetro. La verificación de estos sistemas necesita nuevas metodologías (e. g. orientadas a características, como SAL y MODE, sec. 15.5 en la página 219) y bases de datos de observaciones (como estimaciones de precipitación mediante radar o satélite).

Predecibilidad de la convección. Debido a la naturaleza intrínseca y tremendamente caótica de la convección, la predecibilidad en las escalas convectivas (sec. 22.1 en la página 334) crece más rápidamente que en escalas mayores, como la escala sinóptica. Una consecuencia de este hecho es que para realizar predicciones del tiempo en esas escalas convectivas los SPC serán imprescindibles: sin el enfoque probabilista será muy difícil encontrar otros modos realistas para abordar la predicción del tiempo.

Alcance de este libro. Si los recursos en investigación acompañan a los, casi seguros, avances tecnológicos en satélites y en supercomputación, entonces el proceso de predicción del tiempo y proyección del clima mejorará en todas las escalas, llegando a operar en escalas cada vez más finas. Dentro de esas mejoras, aunque es de esperar una reducción en los errores y las incertidumbres, siempre estará presente un límite en la predecibilidad. Por tanto, todos los conceptos, metodologías y técnicas presentados en este libro seguirán siendo válidos en el futuro y útiles para las próximas generaciones. Así, algunas de las direcciones del futuro ya están trazadas. 


\subsection{Futuro de la predicción del tiempo}

Tendencias de la modelización numérica hacia los SPC y la asimilación de datos. Como ya se menciona en la sección 19.4 en la página 297, en sus planes estratégicos para el periodo 2016-2025, el ECMWF tiene proyectado volcar sus recursos en la predicción por conjuntos, es decir, en su SPC ECENS (sec. 19.3 en la página 293). Como se explica en otro apartado de estas conclusiones, ni que decir tiene que el modelo determinista se seguirá investigando y desarrollando, pues evidentemente es el ingrediente fundamental para el SPC correspondiente, pero ya no será el protagonista de este centro, uno de los centros punteros mundiales. Los centros que disponen de recursos en diversas partes del mundo están apostando por los SPC en uno u otro sentido: EE. UU., Francia, UK, Alemania, Japón, China, etc. (ver cap. 26 en la página 387). En la actualidad (2018), la tendencia en la, así denominada, modelización o predicción numérica del tiempo está orientada a la asimilación de datos (sec. 10.3 en la página 133) y a los SPC (cap. 13 en la página 165), dos grandes ramas que tienen aspectos convergentes.

Predicción probabilista de la precipitación. Las variables meteorológicas de superficie son más complejas que las de altura: la atmósfera es más continua y suave en las alturas que cerca del suelo. En la llamada capa límite planetaria (CLP) la influencia de la orografía, la rugosidad del terreno y el tipo de suelo, son factores críticos que hacen que las variables de superficie sean más complicadas de simular y de predecir. Dentro de las variables de superficie, la precipitación está entre las peores, sin lugar a dudas. Es tremendamente discontinua, es difícil tanto de medir como de simular o predecir, es afectada por la orografía, pero en conjunción con el viento y otros factores que complican el efecto enormemente.

En el capítulo de predicción probabilista abordamos, en un anexo (sec. 27.13 en la página 436), la dificultad añadida que tiene la predicción probabilista de la precipitación cuando vamos aumentando la resolución de los SPC. Resumimos a continuación lo que allí se describe. Los SPC arrojan salidas de un número de miembros que dan precipitaciones en diferentes zonas. La contribución individual a la probabilidad total de precipitación, si el número de miembros es $N$, es de $\frac{1}{N}$. El resultado es que, espacialmente, los SPC tienden a suavizar la señal de precipitación.
Como consecuencia, los predictores operativos, para evaluar las zonas de mayor probabilidad de convección (o precipitación intensa) no buscan directamente en los mapas de probabilidad de $40 \mathrm{~mm} / 6 \mathrm{~h}$, porque éstos dan señales muy débiles en términos de probabilidad. Buscan, inicialmente, en los mapas de probabilidad de 5 ó $10 \mathrm{~mm} / 6 \mathrm{~h}$, que ofrecen señales más claras. Después, con una cierta idea de patrón espacial y de dónde se podrían producir máximos locales, pueden buscarse señales en el mapa de probabilidad de $40 \mathrm{~mm} / 6 \mathrm{~h}$, sabiendo de antemano que una señal en ese mapa tiene una significación diferente. En las situaciones que reúnan los ingredientes sinópticos adecuados, sabemos que la precipitación intensa se va a producir en algún lugar, el problema entonces es dilucidar dónde, y ese punto es extremadamente difícil. Descrito de otro modo, debido a la gran variabilidad espacial de la previsión de precipitación entre diferentes miembros del SPC (sobretodo en la mesoescala o en la escala convectiva), se pueden filtrar los episodios de precipitación intensa y pasar desapercibidos.

Ahora que se están desarrollando SPC en la escala convectiva, se están investigando productos de predicción probabilista nuevos. Por ejemplo, en cada punto de malla puede darse la precipitación del miembro que prevea la máxima precipitación o, si ese campo es excesivo por contener a menudo valores atípicos (outliers), dar la precipitación del percentil 90 de la PDF (sec. 13.6.11 en la página 187) de la precipitación prevista. Productos de este tipo no se deben usar solos y se deberían acompañar, por ejemplo, con otros de probabilidad de umbrales más bajos. Pero, al menos, el predictor tendría idea de las máximas cantidades que se pueden llegar a producir en cada punto, aún a sabiendas que su ubicación puede variar significativamente. Este tipo de mapas son, desde hace mucho, usuales en el NCEP y el NWS de EE. UU., pero en España todavía no está implantado su uso operativo. 13.6.11 en la página 187

\section{Comunicación de la probabilidad y la incertidumbre} en la predicción. La comunicación de la incertidumbre y la probabilidad en la predicción tiene importancia capital, motivando numerosos estudios $[1,6,7$, 10] y, en este libro, el capítulo 40 en la página 615. La sociedad se siente muy implicada en las previsiones meteorológicas. El público general se siente capaz de entender procesos de una complejidad que en el fondo está fuera de su entendimiento y, por otro lado, en esta sociedad del riesgo la prevención de pérdidas socioeconómicas es capital. El ser humano, con su 
tendencia al ahorro cognitivo (generalizar) simplifica mucho la información meteorológica, en el fondo muy compleja, de modo que prefiere una información determinista escueta, aunque sea más susceptible de error, que la riqueza de una información probabilista. El marco de la experiencia de cada individuo hace que la valoración de las predicciones no pueda ser objetiva.

Otro sesgo cognitivo es el efecto de encuadre: según se presente la información podemos interpretar una cosa u otra. Por tanto, la forma de presentar y transmitir la información meteorológica plantea un problema específico [4]. Por todo ello, es crucial para los comunicadores meteorológicos comprender la psicología del usuario final, considerando el significado de la predicción pero también cómo el usuario la «escucha» [6].

Dos estudios del Centro de Investigaciones Sociológicas (CIS) Percepción social de la ciencia y la tecnología (2006 [2]) y Percepción de la meteorología (2011 [3]) profundizan en estos aspectos, como mostramos en las secciones 40.1.3 en la página 617 y 40.1 .4 en la página 618. Dos grandes profesionales de la comunicación, José Miguel ViÑAS y MóNICA LÓPEZ, exponen su visión personal del reto de comunicar probabilidad e incertidumbre, respectivamente, en los medios en general (sec. 40.2 en la página 621) y en la televisión (sec. 40.3 en la página 625).

Fenómenos meteorológicos adversos. Los SPC son herramientas muy poderosas e indispensables para la predicción de fenómenos meteorológicos extremos y de fenómenos meteorológicos adversos (FMA), particularmente en escalas convectivas y especialmente para variables complejas como la intensidad de precipitación o la precipitación acumulada. El plan Meteoalerta (cap. 41 en la página 627) define los umbrales de peligrosidad de cada fenómeno adverso atendiendo a los impactos potenciales en cada zona: amarillo, naranja y rojo.

Con la ayuda de una buena herramienta de ordenador basada en las salidas de un SPC (cap. 35 en la página 569), podemos tener una primer esbozo de aquellas zonas, dentro del dominio de interés (e. g. península ibérica), en las que hay riesgo de los distintos umbrales de aviso, mediante mapas de probabilidad asociados. Para cada variable y cada umbral (amarillo, naranja, rojo) tendremos un mapa de probabilidad asociado.
En este contexto, surge un problema relacionado con los mecanismos de decisión: imaginemos que, en una zona concreta de España definida en Meteoalerta, e. g. Rías Baixas, tenemos las siguientes probabilidades de distintos umbrales de un FMA, e. g. tormentas: $50 \%$ de probabilidad de superar el umbral amarillo, pero simultáneamente $15 \%$ de superar el naranja y, también, $8 \%$ de superar el rojo (una cosa no quita la otra). ¿Cómo puede gestionar el centro de emisión de avisos esta situación? ¿Qué aviso es conveniente emitir? ¿Qué influencia tiene en la decisión el tamaño del área, la extensión geográfica del evento potencialmente previsto? Desde luego, no es una decisión sencilla. Si el área de la zona es importante, lo es aún más el impacto socioeconómico potencial: aquellas zonas más pobladas, con grandes áreas metropolitanas, son las más críticas y donde incluso una probabilidad pequeña debe tenerse en consideración. Por otro lado, la aplicación estricta de las normas Meteoalerta nos permite emitir avisos de un color determinado con una probabilidad asociada. Se argumenta mucho que el usuario no mira esas probabilidades emitidas, pero este argumento se puede discutir.

Si se presta atención a una formación y coordinación rigurosas, el acompañar el aviso de una probabilidad es una poderosísima herramienta para la toma de decisiones, y no debe soslayarse su utilidad. Por último debemos añadir que la experiencia e inteligencia del predictor humano es factor clave en todo el proceso de toma de decisiones en estas situaciones.

Canarias. La modelización de la atmósfera es particularmente difícil en zonas costeras y, sobre todo, en zonas de acusado relieve. Los fenómenos meteorológicos en zonas de elevado gradiente de altitud son difíciles de simular, al igual que las predicciones correspondientes. Como se comenta en diversas ocasiones en el libro, la orografía del modelo o SPC es crucial para el realismo de sus resultados: describir con cierto realismo la topografía del terreno es condición necesaria para conseguir realismo en la meteorología. La orografía de los modelos y SPC ha mejorado ostensiblemente en los últimos años, más o menos a la par con su resolución horizontal. Se ofrece una perspectiva gráfica en el anexo A en la página 927, donde podemos comprobar, como ejemplo, que en los años 80 y 90 el Estrecho de Gibraltar no se tenía en cuenta.

La región española que más dificultades plantea en este aspecto es el archipiélago canario. Con su acusadísimo relieve, con altitudes máximas muy elevadas 
(Teide 3718 metros en Tenerife, Roque de los Muchachos 2426 metros en La Palma, Pico de las Nieves 1956 metros en Gran Canaria, etc.), a escasos kilómetros del océano, convierten a estas islas en un auténtico reto, tanto para modelizadores como para predictores operativos. En 2014 Óscar García Colombo, por aquel entonces Delegado de AEMET en Canarias, manifestaba en una entrevista al diario La Opinión, en Tenerife, que «No hay ordenador capaz de simular el tiempo del Archipiélago» [5]. Hace una década era normal que, de los cuatro puntos de malla del modelo más cercanos al Teide, los cuatro estuviesen en el mar. Obsérvese la «representatividad».

A día de hoy, la situación ha mejorado bastante, pero sigue habiendo aún mucho camino por andar. El modelo HARMONIE-AROME (sec. 20.4 en la página 310) se integra en un dominio específico para Canarias, con una resolución horizontal de $2.5 \mathrm{~km}$; pueden verse algunos resultados en el capítulo 56 en la página 839. El SPC AEMET- $\gamma$ SREPS (cap. 22 en la página 333), se integra a esa misma resolución, en la actualidad en Península y Baleares y pronto en Canarias. Se tiene idea de que para obtener resultados razonablemente realistas hay que integrar modelos a resoluciones de $1 \mathrm{~km}$ o, a ser posible, $500 \mathrm{~m}$. Ya se han hecho algunas pruebas con resultados prometedores, aunque aún queda un tiempo para poner en marcha algo sistemático.

Papel del predictor en el futuro. Existe un desfase entre el desarrollo de herramientas para la predicción operativa y la propia operatividad, debido a cuestiones de organización y de poco equilibrio en el reparto de los recursos. Este desfase debería ser cada vez menor. Por otro lado, se está vislumbrando un camino hacia lo que denominamos predicción sin costuras o sin discontinuidades (seamless): una cadena de modelos y SPC para cada escala y plazo (van relacionados), pero que enganchen unos con otros sin saltos bruscos. El término seamless, aplicado a la modelización climática, también se refiere a un mismo modelo o SPC utilizado para diferentes escalas/plazos, permitiendo, por ejemplo, que la posibilidad de verificación en escalas o plazos menores, se pueda aplicar aportando mejoras en las predicciones/proyecciones en escalas o plazos mayores (ver sec. 28.1.8 en la página 458).

Podemos, además, ampliar esa concepción extendiéndola también a la observación en tiempo real. Así, desde la observación pasaríamos al nowcasting, luego al muy corto plazo, al corto, al medio y al largo plazo, con toda la información integrada en la misma plataforma y sin saltos aparentes entre distintos plazos. En el ámbito de Meteoalerta, en la emisión de avisos puede mejorarse todavía mucho la difusión de los mismos, en tiempo y en contenidos, mejorando asimismo la coordinación con protección civil. Por ejemplo, numerosos usuarios especializados reciben información en tiempo real de la Dirección General de Tráfico (DGT) en formato visual e interactivo, pero la información meteorológica les sigue llegando por fax en formatos muy escuetos. Es preocupante que, con los recursos disponibles y el esfuerzo dedicado a hacer predicciones de calidad, en ocasiones lo que llegue al usuario sea una información muy filtrada y degradada.

En la sección 4.4.6 en la página 45 hemos emitido conclusiones acerca del futuro valor añadido del predictor. Es de suponer que los modelos y SPC seguirán mejorando, de modo que el predictor tendrá progresivamente menos margen para la corrección de sesgos sistemáticos (hace 15 años un predictor mejoraba enormemente las salidas de los modelos, ahora ya no tanto), aunque en aspectos como los fenómenos adversos, es probable que todavía pueda seguir aportando mucho, sin olvidarnos de la predicción de la convección y de la vigilancia meteorológica. En cualquier caso, el valor añadido evolucionará, aunque todavía en direcciones que no podemos vislumbrar con claridad, encaminándonos hacia esa «predicción operativa 2.0». El margen actual de mejora en los centros operativos no está en la automatización de la predicción, sino en muchos otros aspectos.

Las nuevas tecnologías de la información y las comunicaciones son un primer referente de mejora. El plan Meteoalerta podría estar disponible en los teléfonos móviles, por ejemplo.

El papel de las redes sociales para la distribución de la información, así como para la obtención de nuevos datos (e. g. SINOBAS, el sistema de AEMET de recogida de información ciudadana https : //sinobas.aemet.es/), es crucial, y una parte del personal de meteorología puede dedicarse a la supervisión. Los modelos conceptuales, fundamentales para la escala sinóptica, no están tan presentes en la predicción operativa para la mesoescala y aún menos para la escala convectiva, pero esos modelos conceptuales existen: falta ahí una labor de formación que se ha debilitado bastante. Podrían automatizarse algunas tareas mecánicas que continúan haciendo los predictores por razones diversas, principalmente por falta de 
recursos.

El papel de relevancia creciente de la predicción probabilista, aunque puede retrasarse por la renuencia de muchos usuarios, ofrecerá a los predictores la oportunidad de orientar una parte de sus tareas hacia asesoramiento técnico y consultoría sobre la interpretación y uso de los productos probabilistas [8], tanto para usuarios especializados (e. g. protección civil, aviación, etc.) como generales. En esa línea los predictores podrían participar activamente en el diseño de productos específicos. La formación de los usuarios es un aspecto de vital importancia en este sentido, y los servicios meteorológicos debemos poner un poco de presión en el uso de la predicción probabilista, dado que es fiable y dado que es rentable.

Otro importante ámbito de cultivo del valor añadido es la verificación de los modelos y SPC, así como la monitorización de los mismos y de herramientas derivadas. Existen, por ejemplo, herramientas de seguimiento y extrapolación de fenómenos convectivos, que requieren esa monitorización.

Es importante, tanto en el momento actual como en la evolución próxima, ir revisando el estado del arte en predicción, sondear el tipo de servicios meteorológicos demandados por la sociedad, reclamando así la importancia de nuestra profesión. Es capital evitar la automatización a ciegas, motivada a menudo por la presión externa de reducción de personal. Aquellos que apuestan por la automatización completa del proceso predictivo (apuesta que se lleva haciendo desde los años 70, por parte de ciertos sectores) demuestran estar lejos de la realidad de dicho proceso. Intentemos que, en el debate de estos temas sobre el futuro de la predicción, tengan voz todos los eslabones de la cadena. Así lo esperamos. 


\subsection{Referencias}

[1] Abraham, Shymali y col. "Do locationspecific forecasts pose a new challenge for communicating uncertainty?" En: Meteorological Applications 22.3 (2015), páginas 554-562. DOI: 10.1002 /met . 1487 (citado en página 921).

[2] Centro de Investigaciones SociolóGICAS. Percepción social de la ciencia y la tecnología. Informe técnico. 2006, página 7056 (citado en página 922).

[3] Centro de Investigaciones SociolóGICAS. Percepción de la meteorología. Informe técnico. Centro de Investigaciones Sociológicas, 2011, página 2959. URL: http: / / www . cis . es / cis / opencm / ES / 1 _ encuestas / estudios / ver . jsp? estudio=12764 (citado en página 922).

[4] Crespo, Eduardo, Revilla, Juan Carlos y Elizaga, Fermín. "Meteorología y sociedad: uso y evaluación de la información meteorológica/Meteorology and society: use and evaluation of meteorological information". En: Comunicación y Sociedad 27.2 (2014), página 169 (citado en página 922).

[5] JimÉnEZ, Juanjo. Óscar García: No hay ordenador capaz de simular el tiempo del Archipiélago. Tenerife, ago. de 2013. URL: http://www . laopinion.es/sociedad/ 2013/08/16/hay-ordenador - mundocapaz-simular/493152.html (citado en página 923).
[6] Joslyn, Susan y SAVelli, Sonia. "Communicating forecast uncertainty: Public perception of weather forecast uncertainty". En: Meteorological Applications 17.2 (2010), páginas 180-195. DOI: $10.1002 /$ met . 190 (citado en páginas 921, 922).

[7] Kootval, H. "Guidelines on communicating forecast uncertainty". En: World Meteorological Organization/Technical Document 1422 (2008) (citado en página 921).

[8] Novak, David R, BRIght, David R y BRENNAN, Michael J. "Operational forecaster uncertainty needs and future roles". En: Weather and Forecasting 23.6 (2008), páginas 1069-1084 (citado en página 924).

[9] Scotт, Alwyn C. The nonlinear universe: chaos, emergence, life. Springer Science $\&$ Business Media, 2007 (citado en página 918).

[10] TAYlor, Andrea L, Dessai, Suraje y BRUIN, Wändi Bruine de. "Communicating uncertainty in seasonal and interannual climate forecasts in Europe". En: Phil. Trans. R. Soc. A 373.2055 (2015). DOI: 10.1098/ rsta.2014.0454 (citado en página 921).

[11] WIENER, Norbert. "Nonlinear prediction and dynamics". En: Proc. 3rd Berkeley Sympos. Math. Stat. and Prob. Volumen 3. 1956, páginas 247-252 (citado en página 918). 